\title{
Hepatic stellate cells promote tumor progression by enhancement of immunosuppressive cells in an orthotopic liver tumor mouse model
}

\author{
Wenxiu Zhao ${ }^{1}$ Lei Zhang ${ }^{1}$ Yaping Xu, Zhengqi Zhang, Guangli Ren, Kai Tang, Penghao Kuang, \\ Bixing Zhao, Zhenyu Yin and Xiaomin Wang
}

The immunosuppressive properties of hepatic stellate cells (HSCs) contribute to the occurrence and development of hepatocellular carcinoma (HCC). The accumulation of cells with immune suppressive activities, such as myeloid-derived suppressor cells (MDSCs) and regulatory T cells (Tregs) is a key mechanism for tumor immune evasion. However, the impact of HSCs on immune cell populations in tumor-bearing hosts is unclear. In this study, we established an orthotopic liver tumor mouse model for studying the complex tumor-host interactions in HCC. The activated HSCs promoted HCC growth not only induced tumor angiogenesis and lymphangiogenesis, but also significantly increased the suppressive immune cell population of Tregs and MDSCs in the spleen, bone marrow, and tumor tissues of the tumor-bearing mice. Murine HCC cell line H22-activated HSCs also expanded the expression of Tregs and MDSCs in vitro. In conclusion, our study suggests a novel role for HSCs in the HCC microenvironment. HSCs can promote HCC progression by enhancement of the immunosuppressive cell population. Targeting HSCs, which is a new concept in adjuvant immunotherapy, may be introduced in the near future to improve the outcome of patients with HCC.

Laboratory Investigation (2014) 94, 182-191; doi:10.1038/labinvest.2013.139; published online 2 December 2013

KEYWORDS: hepatic stellate cells; hepatocellular carcinoma; myeloid-derived suppressor cells; regulatory T cells

Hepatocellular carcinoma (HCC) is one of the most common human cancers worldwide. Recently, it was reported that the biologic behaviors of HCC are associated with a unique immune response signature of the liver microenvironment. ${ }^{1}$

The liver is a unique organ in which induction of tolerance may be favored over the induction of immunity. Hepatic tolerance has been characterized by relatively low rates of liver allograft rejection. However, hepatocyte transplantation resulted in rejection, which suggested that liver nonparenchymal cells may contribute to an immune-tolerant environment to protect hepatocytes from immune attack. Qian and colleges first reported that hepatic stellate cells (HSCs) have a strong immunomodulatory activity and can prolong islet allografts survival. ${ }^{2,3}$

Accumulating evidence supports that tumors have the ability to subvert an immune attack and tumor immune evasion has been recognized as a 'Hallmark of Cancer." The mechanisms by which tumor cells evade the host immune system have been investigated. One mechanism of tumor immune evasion is the accumulation of cells with immunosuppressive activities at both the tumor site and different primary and secondary lymphoid organs. These cells include regulatory $\mathrm{T}$ cells (Tregs) and myeloid-derived suppressor cells (MDSCs). Several studies have revealed the importance role of Tregs and MDSCs in cancer progression. ${ }^{5,6}$ Tregs, a functionally unique subset of $\mathrm{T}$ cells, can suppress effective antitumor immune responses. ${ }^{7}$ The increase of Tregs in the peripheral blood and tumor sites has been reported in HCC patients. ${ }^{8-10}$ Another important immunosuppressive population, MDSCs, has been shown to be upregulated in HCC patients. ${ }^{11}$ MDSCs are a heterogeneous population of myeloid cells, and are commonly defined by the markers, CD11b and Gr-1 (Ly6-C/G), in mouse models. MDSCs can be detected in the blood, bone marrow, spleen, lymph nodes, and tumor microenvironment where they suppress the specific cellular response to cancer cells. ${ }^{12}$

In our previous study, we observed that HSCs can create an immunosuppressive environment that promotes HCC growth in a subcutaneous transplantation of HCC model. ${ }^{13}$

Department of Hepatobiliary Surgery, Zhongshan Hospital, Xiamen University, Fujian Provincial Key Laboratory of chronic liver disease and hepatocellular carcinoma (Xiamen University Affiliated Zhongshan Hospital), Xiamen, China

Correspondence: Professor X Wang or Z Yin, Department of Hepatobiliary Surgery, Zhongshan Hospital, Xiamen University, Fujian Provincial Key Laboratory of chronic liver disease and hepatocellular carcinoma (Xiamen University Affiliated Zhongshan Hospital), South Hubin Road \#209, Xiamen 361004, China.

E-mail: wxm2203@xmu.edu.cn or yinzy@xmu.edu.cn

${ }^{1}$ These authors contributed equally to this study.

Received 28 March 2013; revised 15 September 2013; accepted 29 September 2013 
However, the relationship between HSCs and the immunosuppressive cells in the HCC microenvironment are unclear. In this study, we established an orthotopic liver tumor mouse model for studying the complex tumor-host interactions in HCC. Our data have shown that HSCs can promote HCC progression by enhancement of the immunosuppressive cell population. Our studies provide important insights into understanding the novel mechanism by which HSCs promoting the HCC development.

\section{MATERIALS AND METHODS Isolation and Culture of HSCs}

HSCs were isolated from BALB/c mouse livers as previously described. ${ }^{13}$ The isolated HSCs were cultured in RPMI1640 medium (Hyclone) supplemented with 10\% heat-inactivated fetal bovine serum (Gibco), $100 \mathrm{U} / \mathrm{ml}$ penicillin and $100 \mu \mathrm{g} /$ $\mathrm{ml}$ streptomycin (RPMI1640 complete medium) in a humidified atmosphere with $5 \% \mathrm{CO}_{2}$ at $37^{\circ} \mathrm{C}$. Cells were used at passages $4-10$ for the experiments. Assessment of activation was performed by $\alpha$-SMA staining of cells. ${ }^{13,14}$

\section{Cell Lines}

The mouse hepatoma cell line $\mathrm{H} 22$ was purchased from Shanghai Cell Bank, Chinese Academy of Sciences, and maintained in RPMI1640 complete medium.

\section{Culture of Dendritic Cells (DCs)}

The culture of DCs was performed as previously described. ${ }^{14}$

\section{In Situ Hepatic Tumor Model}

C57BL/6(H-2b, haplotype) and BALB/c (H-2d, haplotype) mice were purchased from the National Rodent Laboratory Animal Resources, Shanghai branch. Adult male animals, aged 8-12 weeks, were used. The HCC model was created by the direct intrahepatic injection of mouse hepatoma cells. The mice were anesthetized and a midline incision was made to expose the liver. The $\mathrm{H} 22$ cells alone $\left(1 \times 10^{6}\right.$ cells originating from the $\mathrm{BALB} / \mathrm{c}$ mice, $\mathrm{H}-2 \mathrm{~d}$ haplotype) or plus $2 \times 10^{5}$ HSCs or NIH3T3 were resuspended in 30-50 $\mu$ l of PBS $(n=5)$. The cells were then slowly injected under the hepatic capsule into the upper left lobe of the liver using a $28-G$ needle. A pale, whitish color could be observed at the point of injection under the hepatic capsule. Gentle compression was applied for $15 \mathrm{~s}$ with a cotton applicator to avoid bleeding and reflux of the cells. The abdomen was closed with a 5-0 silk suture. The mice were observed for $2-3 \mathrm{~h}$ and then returned to the housing facilities.

All the experimental protocols were reviewed and approved by our institutional review board. All animal experimental protocols were approved by the Institutional Animal Care and Use Committee of Xiamen University.

\section{Detection of Tregs}

To evaluate the impact of HSCs on Tregs, the mixed lymphocyte reaction (MLR) was performed in 24-well microtiter plates. Briefly, nylon wool-eluted C57BL/6T cells $\left(1 \times 10^{6}\right)$ were cultured with DCs at a T-cell/DC ratio of 10:1 for 3 days. Mitomycin C-treated $(40 \mu \mathrm{g} / \mathrm{ml})$ HSCs or H22-activated HSCs were added at the beginning of the MLR culture (T-cell/HSC ratio 40:1). To detect the Tregs, cells were harvested and stained with FITC-CD4 and PE-Foxp3 antibodies (all from eBioscience). The cells were then analyzed by flow cytometry. To obtain the H22-activated HSCs, cells were cultured using 3- $\mu \mathrm{m}$ cell culture inserts that kept both cell populations separate. HSCs were seeded into 6-well plates in RPMI 1640 medium supplemented with 10\% fetal bovine serum for $12 \mathrm{~h}$. Then H22 cells were seeded onto the insert and co-cultured with HSCs for an additional $24 \mathrm{~h}$ (H22/HSC ratio $1: 5)$.

\section{Detection of MDSCs}

To determine the effect of HSCs on DCs propagation, BM cells were isolated from the femurs of $\mathrm{BLAB} / \mathrm{c} /$ mice and cultured in the presence of GM-CSF and IL- 4 for 6 days. Then, cells were exposed to LPS at $1 \mu \mathrm{g} / \mathrm{ml}$ for the last $18 \mathrm{~h}$ of culture. HSCs were added at the beginning of the culture at a HSC/DC ratio of 1:40. Cells were harvested and stained with PE-Gr-1 and FITC-CD11b (all from eBioscience), and analyzed by flow cytometry.

\section{Isolation of the Intrahepatic Leukocytes (IHLs)}

After perfusion with PBS and $0.01 \%$ collagenase, the liver was removed and peritumor tissue $(n=5)$ was collected and mixed together per group. Then peritumor tissues were homogenized and digested for $30 \mathrm{~min}$ at $37^{\circ} \mathrm{C}$ using $0.01 \%$ collagenase. Cells were washed with RPMI1640 and then underlaid with $24 \%$ metrizamide in PBS. After centrifugation for $20 \mathrm{~min}$ at $1500 \mathrm{~g}$, IHLs were harvested from the mediummetrizamide interphase. Erythrocytes were lysed with Red Blood Cell Lysis Buffer (Beyotime) and subsequently washed in RPMI1640 complete medium. The purity of the isolated cells was confirmed by staining with CD45 (eBioscience), a common leukocyte antigen.

\section{Flow Cytometry}

Splenocytes were isolated from the spleen of tumors-bearing mice by disaggregation into $10 \mathrm{ml}$ RPMI1640 complete medium. Erythrocytes were lysed with Red Blood Cell Lysis Buffer (Beyotime). $1 \times 10^{6}$ splenocytes were incubated with PE-conjugated CD3, Gr-1, Foxp3, and FITC-conjugated CD4, CD8, CD11b (all from eBioscience).The fluorescently labeled cells were analyzed on a flow cytometry (Beckman, USA) using the CELLQuest Pro software. The appropriate isotype control antibodies were used.

\section{Immunofluorescence}

Immediately after dissection, tumor samples were embedded in OCT compound, snap-frozen, and stored at $-80^{\circ} \mathrm{C}$ until use. Five-micrometer cryosections were cut, air-dried, acetone-fixed, blocked with $10 \%$ normal goat serum (30 min, 
Zymed). To detect MDSCs expression, slides were incubated with antibody Alexflour 488-Gr-1 (dilution 1:100; Invitrogen) for $60 \mathrm{~min}$ at $37^{\circ} \mathrm{C}$. After incubation, the slides were washed three times in TBS-T for $5 \mathrm{~min}$, and then incubated with antibodies PE-CD11b (dilution 1:100; BD Pharmingen) for $60 \mathrm{~min}$ at $37^{\circ} \mathrm{C}$. To detect Tregs expression and microvessel density, sections were incubated with rat anti-mouse foxp3 or CD31 (dilution 1:100; BD Pharmingen) for $60 \mathrm{~min}$ at $37^{\circ} \mathrm{C}$, then add 1:400 diluted Alexflour 488-conjugated anti-rat secondary antibody (Invitrogen) for $30 \mathrm{~min}$ at $37^{\circ} \mathrm{C}$ in a humidified chamber. To evaluate lymphatic-vessel density in tumors, LYVE-1 was identified in cryostat sections using Alexflour 488 LYVE-1 (eBioscience). After incubation, the slides were washed three times in TBS-T for $5 \mathrm{~min}$. Sections were stained with $0.5 \mu \mathrm{g} / \mathrm{ml}$ of DAPI stain for $2 \mathrm{~min}$, rinsed briefly in TBS-T, and then mounted using Prolong anti-fade mounting medium and visualized and photographed using a Leica DM2500 microscope.

\section{Quantification of Immunostaining in Tumors}

The immunostaining of Tregs and MDSCs was quantified by counting positive cells and total number of cells at five arbitrarily selected fields from each group at $\times 400$ magnification. And data are presented as number of positive or double positive cells per high-power fields (HPFs). For the lymphatic vessel densities, LYVE-1 + and DAPI + area were measured using Image J software and presented as percentage per DAPI + area. For the blood vessel densities, slides were examined at an original magnification of $\times 4$. Five 'hot spots' (areas with the highest microvessel concentration) from each slide were identified and these areas were photographed at an original magnification of $\times 20$. The area of this histological field was $708588 \mu \mathrm{m}^{2}$. Two independent observers counted the number of microvessels in the histological field. The number of blood vessels was presented as blood vessel numbers of $\mathrm{mm}^{2}$.

\section{5-(and -6)-Carboxyfluorescein Diacetate Succinimidyl Ester (CFSE) Labeling for HSCs}

To detect the orthotopic co-infusion of HSCs and HCC, HSCs were stained with the intracellular fluorescent dye CFSE before injection using CFSE Cell Proliferation Assay and Tracking Kit (Beyotime) according to the manufacturer's instructions. Ten days after injection, the mouse was killed. The liver lobe with tumor was removed, placed into optimal cutting temperature and immediately sectioned without fixation and analyzed by fluorescent microscopy.

\section{Statistical analysis}

The data were analyzed using the SPSS software (version 13.0). The results are expressed as means \pm s.d. Statistical analyses were performed using a one-way ANOVA and Student's $t$-test. The significance level was set at 0.05 .

\section{RESULTS}

\section{Activated HSCs Support Tumor Growth in a Murine Orthotopic Transplantation Liver Cancer Model}

The orthotopic implantation of tumor cells in immunocompetent mice is representative of naturally occurring HCC and is preferable for studying the complex tumor-host interactions for HCC. To investigate the effects of HSCs on HCC cells in vivo, a murine HCC model was established by orthotopic transplantation of $\mathrm{H} 22$ cells alone $\left(1 \times 10^{6}\right.$ cells/ mouse) or $\mathrm{H} 22$ combination with HSCs $\left(2 \times 10^{5}\right.$ cells/ mouse) into $\mathrm{BALB} / \mathrm{c}$ mice $(n=5)$. The tumors arising from the group of mice that received the $\mathrm{H} 22$ cells combined with the HSCs were significantly larger than the tumors form the group of mice that received H22 cells only (Figure 1a). The average volume of the tumors from the mice that received the mixed cell populations was $300 \%$ of the control groups (Figure 1b). Our findings are consistent with our previous reports, which showed that activated HSCs promoted HCC growth in a murine model bearing subcutaneous tumors. ${ }^{13}$

To further confirm the effect of HSCs on HCC development, murine NIH3T3 cells were used as control fibroblast line. The mixture of $1 \times 10^{6} \mathrm{H} 22$ cells, with or without $2 \times 10^{5}$ cells HSCs or NIH3T3 was injected into the liver lobe $(n=5)$. As shown in Figure 1c, co-injection of HSCs contributed to the generation of larger tumors in contrast to the co-injection of NIH3T3, which did not enhance tumor mass growth. Furthermore, similar results were also observed in subcutaneous HCC mice model(data not shown).Taken together, these data suggest that it is HSCs but not NIH3T3 cells that promote HCC development and implantation of HSCs is sufficient to enhance HCC development in vivo.

To detect the orthotopic co-infusion of HSCs and H22, HSCs were stained with CFSE before injection. As the results shown, the CFSE-labeling HSCs infiltrated the stroma of HCC, localizing around tumor capsule (Figure 1d).

\section{Activated HSCs Promoted Tumor Angiogenesis and Lymphangiogenesis}

Experimental and clinical data indicated that the pathogenesis of HCC growth and metastasis was linked to the angiogenesis and proliferation of abnormal vessels. ${ }^{15}$ To further explore the tumor-promoting effect of HSCs, tumor angiogenesis and lymphangiogenesis were analyzed in tumors derived from HSCs co-transplanted group and the H22 only group. Vascular density, as determined by CD31-positive area, was significantly higher in the tumors derived from the mixture of HSCs and $\mathrm{H} 22$ cells (Figures $2 \mathrm{a}$ and $\mathrm{b}$, $P<0.01$ ).

The recent identification of novel lymphatic markers can accurately discriminate the lymphatic vessels from the blood vessels in tissue sections. Of these, LYVE-1 has been most commonly used in studies to assess the significance of the lymphatic vessel density/lymphatic area. ${ }^{16}$ To assess the growth and the patterning of lymphatic vasculature, an immunofluorescence analysis was performed with anti-LYVE-1. 
a
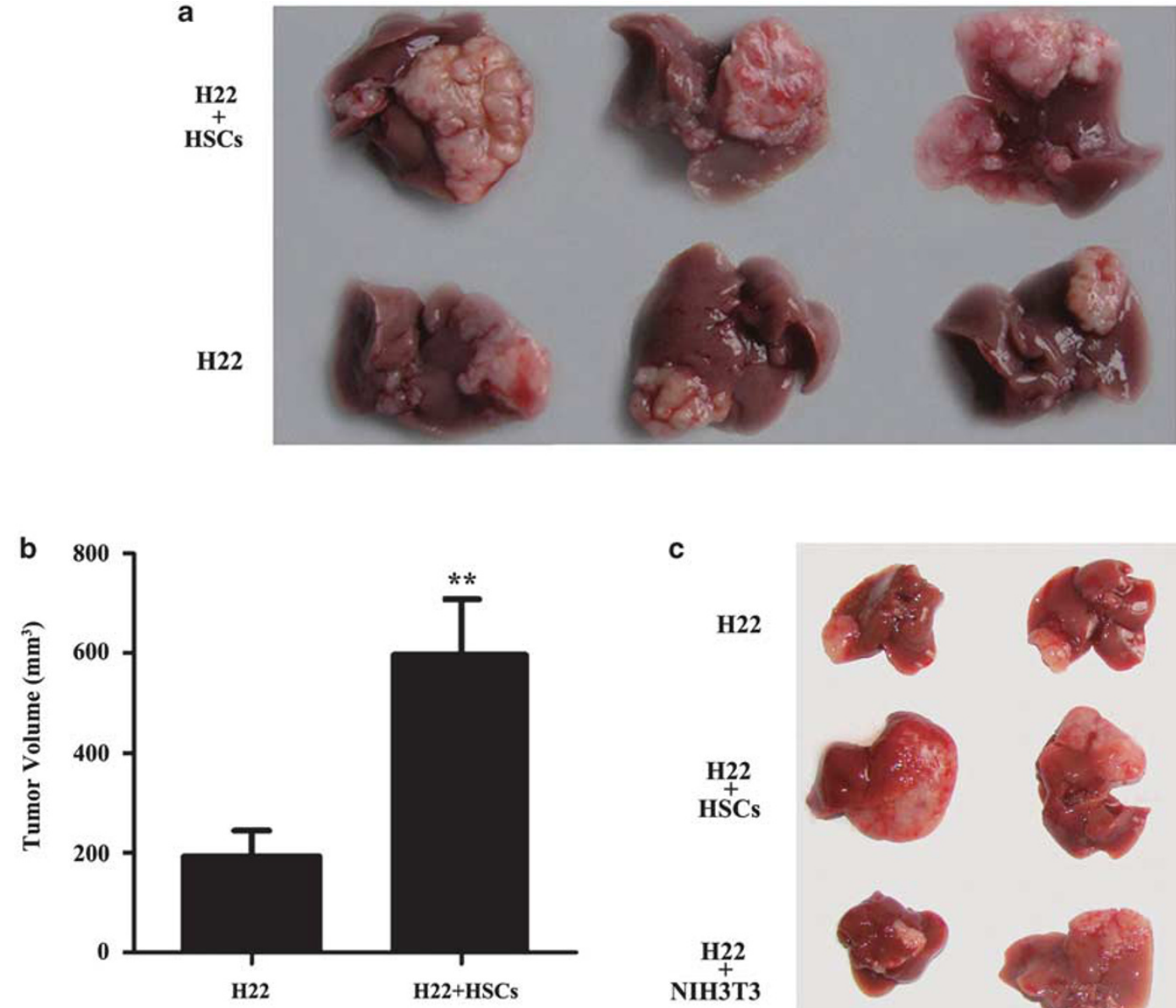

C

$\mathbf{H} 22$

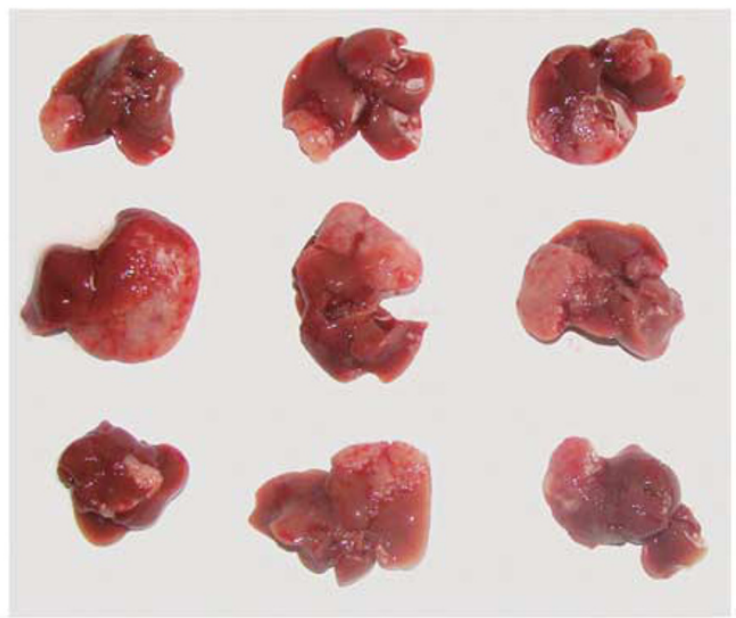

d

CFSE-HSCS

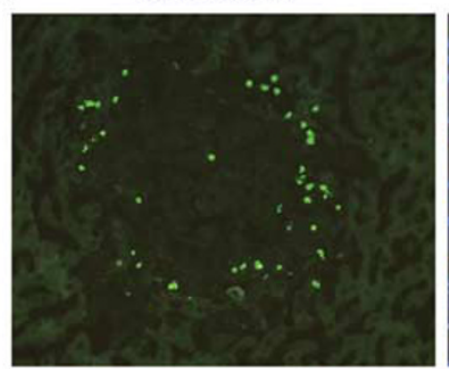

DAPI

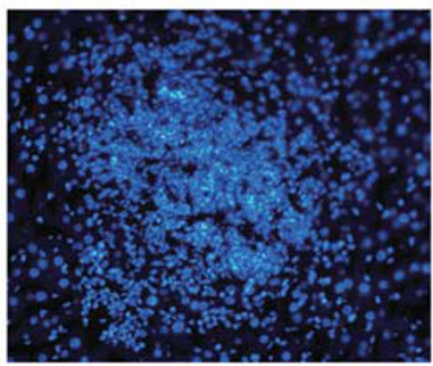

Overlay

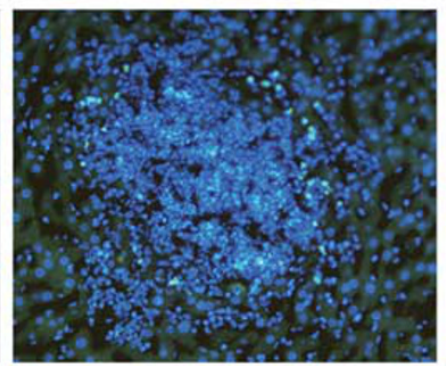

Figure 1 Hepatic stellate cells (HSCs) promoted the growth of hepatocellular carcinoma (HCC) in an orthotopic HCC model. BALB/c mice were injected intrahepatically with $\mathrm{H} 22$ cells or $\mathrm{H} 22$ cells plus $\mathrm{HSCs}(n=5)$. Ten days after implantation of the cells, the mice were killed. Representative liver tumors resected from the mice were shown (a) and the tumor volume (b). The mixture of $1 \times 10^{6} \mathrm{H} 22$ cells, with or without $2 \times 10^{5} \mathrm{HSCs}$ or NIH3T3 cells, was injected into the liver lobe. Representative images from each group were shown (c). (d) Tumor cryosections were observed using fluorescence microscopy for CFSE-labeled HSCs. ${ }^{*} P<0.01$.

As shown in Figure 2c, the lymphatic vessels were more abundant in the HSC co-transplanted group. A quantitative analysis confirmed that the percentage of LYVE-1-positive area was significantly higher in the HSC co-transplanted group (Figure 2d).
Co-transplanted HSCs Reduced the Expression of T-cell Subsets in the Spleen and Expanded the Proportion of Treg Cell Population

$\mathrm{CD}^{+}$and $\mathrm{CD}^{+}{ }^{+} \mathrm{T}$ cells are considered the primary mediators of immunotherapy, and the proportion of T-cell 
a

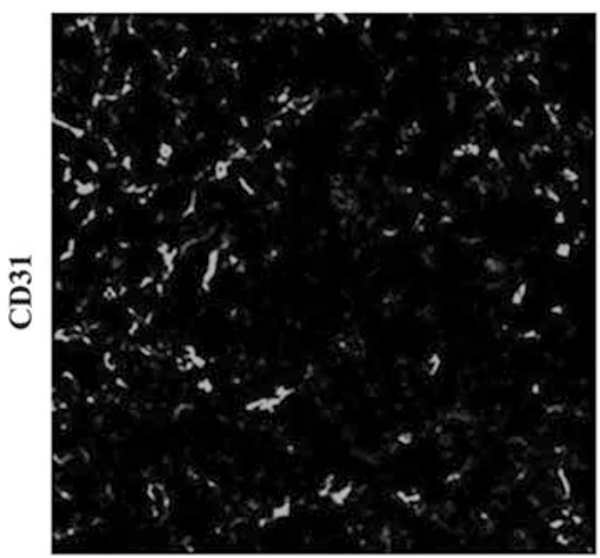

C

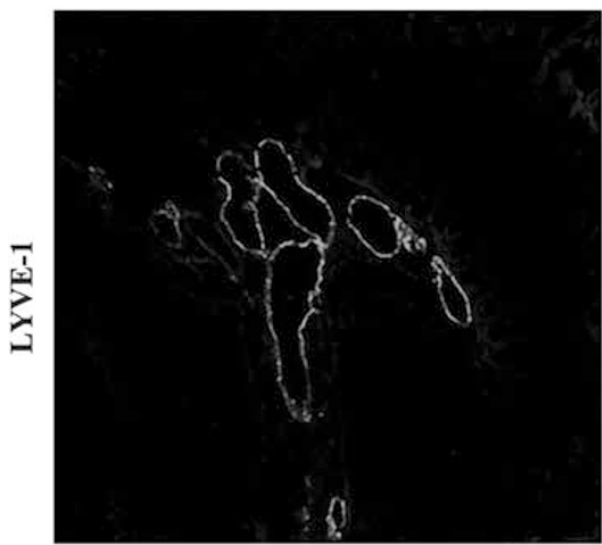

H22

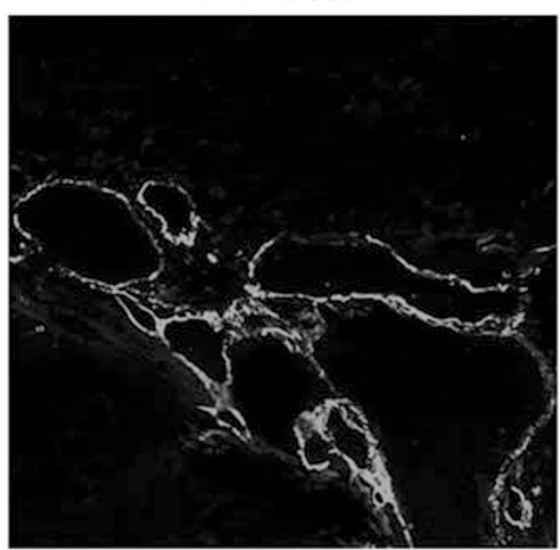

b
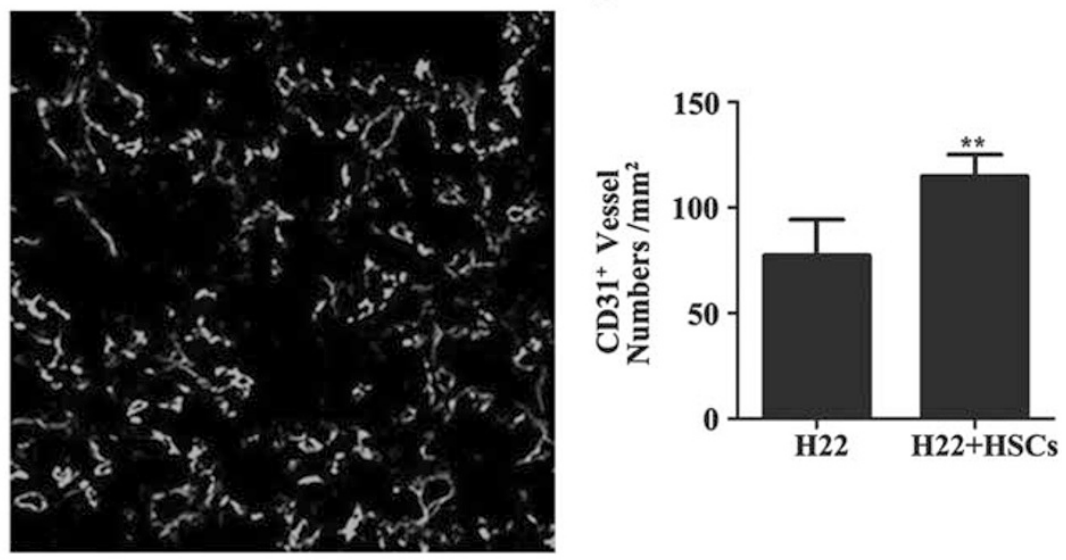

d

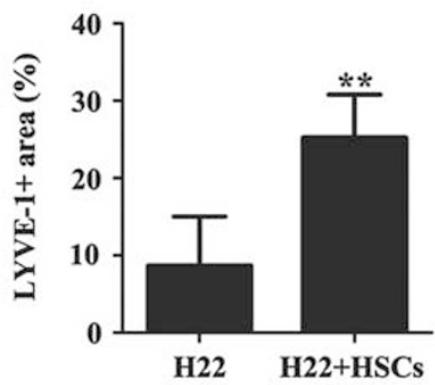

Figure 2 Activated hepatic stellate cells (HSCs) promoted tumor angiogenesis and lymphangiogenesis. The tumor cryostat sections were processed for immunofluorescence staining with anti-CD31 and anti-LYVE-1 antibodies. (a) cd31-positive blood vessels. (b) The mean of blood vessel numbers per $\mathrm{mm}^{2}$ is reported. (c) Lyve-1-positive lymphatic vessels. (d) Quantitative data for lymphatic vessel densities are shown as percentage of LYVE-1 + area per DAPI + area. The sections were analyzed at $\times 200$ magnification and the data are expressed as the mean \pm s.d. ${ }^{* *} P<0.01$.

subsets in the spleen have a critical role in tumor rejection in orthotopic models. ${ }^{17}$ To investigate the immunological basis for tumor growth following the injection with HSCs, we quantified the T-cell-mediated immune response in the spleen using flow cytometry analysis. The results showed that the CD3/4 T cells were downregulated from $24.6 \%$ in the $\mathrm{H} 22$ only group to $16.2 \%$ in the H22 plus HSCs group (Figure 3a, upper, $P<0.05, n=5$ ). Meanwhile, the HSCs markedly decreased the expression of CD3/8 $\mathrm{T}$ cells from 11.8 to $5.7 \%$ (Figure 3 a, lower, $P<0.01, n=5$ ).

The important immunosuppressive cells, Tregs, have been reported to be elevated in HCC patients. ${ }^{8-10}$ To investigate whether HSCs could also induce the expansion of Tregs, $\mathrm{CD} 4{ }^{+}$Foxp $3^{+}$Tregs in the spleens of tumor-bearing mice were examined by flow cytometry. As shown in Figure 3b, the HSC co-transplanted group had a higher percentage of Foxp3 expression in $\mathrm{CD} 4{ }^{+} \mathrm{T}$ cells $(P<0.001, n=5)$.

\section{Co-transplanted HSCs Induced the Expansion of MDSCs in the Spleen and Bone Marrow}

MDSCs are a heterogeneous population of cells that have been shown to be involved in the regulation of the immune response to increase in several cancers, including $\mathrm{HCC}^{11}$ and so on. ${ }^{12}$ The induction of MDSCs was regarded as one of the mechanisms of the immunoregulatory activity of HSCs. ${ }^{18}$ To confirm whether co-transplantation of HSCs could induce MDSC expansion in the mouse HCC model, the MDSC populations in the spleen and bone marrow were analyzed by flow cytometry. As shown in Figure 4a, the presence of $\mathrm{CD} 11 \mathrm{~b}^{+} \mathrm{Gr}-1^{+}$MDSCs was significantly increased in the bone marrow of the HSC co-transplanted group $(P<0.05$, $n=5$ ). The same results were observed in the spleen, where the population of MDSCs was $6.2 \%$ in the control group and $15.5 \%$ in the HSC co-transplanted group (Figures $4 \mathrm{~b}$, $P<0.05, n=5)$.

\section{Co-transplanted HSCs Induced the Expansion of Suppressive Cells in the Tumor Environment}

The above results demonstrated that HSCs could induce the expansion of immunosuppressive cells in the spleen and bone marrow, which prompted us to evaluate whether HSCs also have the same role in the HCC microenvironment. To test this hypothesis, we performed an immunohistochemical assay in the liver tissue. As shown in Figures $5 \mathrm{a}$ and b, 

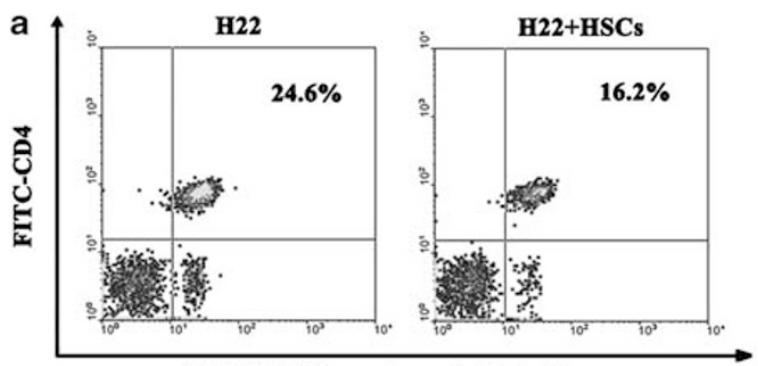

PE-CD3(Lymphocyte Gated)
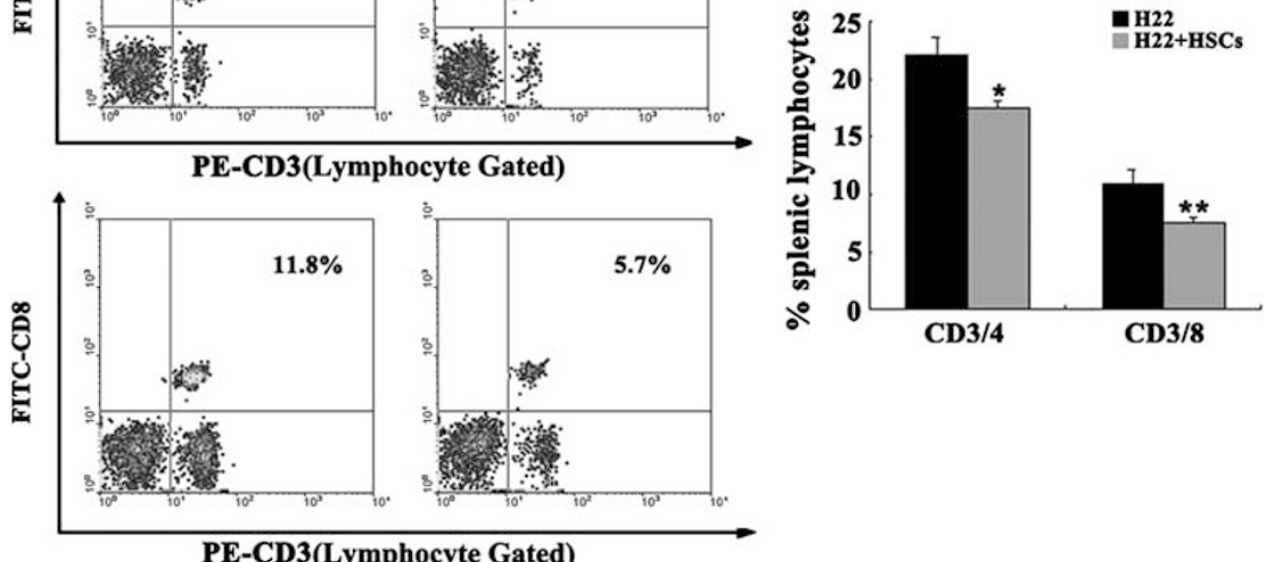

b

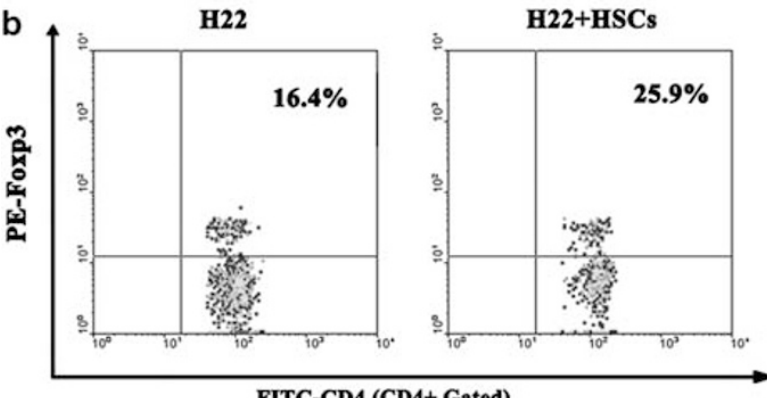

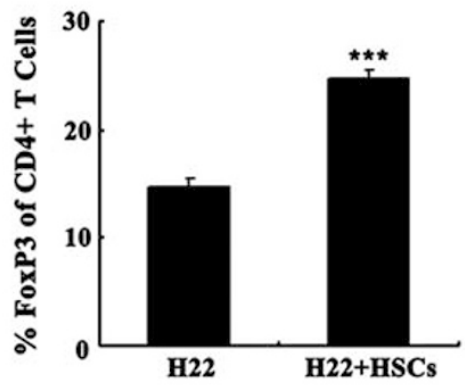

Figure 3 The effect of hepatic stellate cells (HSCs) on the expansion of T cells and its subsets in the spleen from tumor-bearing mice $(n=5)$. (a) The splenocytes were double-stained with fluorescently labeled CD3 and CD4 mAbs or CD3 and CD8 mAbs and gated on the lymphocyte cells. (b) The splenocytes were double-stained with CD4 and Foxp3 mAbs for flow cytometric analysis and gated on the CD4 ${ }^{+}$cell populations. The data are expressed as the mean \pm s.d. ${ }^{*} P<0.05,{ }^{*} P<0.01,{ }^{* * *} P<0.001$.

compared with the $\mathrm{H} 22$ only group, the HSC co-transplanted group showed an abundance of Foxp3-stained and Gr-1 ${ }^{+}$ $\mathrm{CD}_{11 \mathrm{~b}^{+}}$double-stained cells in the mesenchymal areas of the tumor. However, there were very low levels of Foxp3 staining or Gr-1 and CD11b double staining in tumor area (data not shown). We next isolated IHLs from peritumoral tissues and analyzed these cells by flow cytometry. As shown in Figures $5 \mathrm{c}$ and $\mathrm{d}$, the HSC co-transplanted group contained a higher percentage of Tregs and MDSCs than the H22 only group.

\section{H22-Activated HSCs Induced the Expansion of Tregs and MDSCs In Vitro}

To understand the mechanisms behind the immune suppression of HSC co-transplantation in vivo, we examined the effect of tumor-activated HSCs on the expansion of immunosuppressive cells in vitro. First, HSCs were cocultured with the hepatoma cell line $\mathrm{H} 22$, using a cell culture insert system for $24 \mathrm{~h}$ and then collected as tumoractivated HSCs. To identify whether the proliferation of Tregs is mediated through HSCs, H22-activated HSCs or activated
HSCs were added to the MLR culture at a HSC/DC/T ratio of 1:2:20 for 3 days. As shown in Figure 6a, CD ${ }^{+} \mathrm{Foxp}^{+}$cell populations were upregulated in the coculture with the activated HSCs. Furthermore, the increasing trend became more obvious in the coculture with the H22-activated HSCs.

We have shown that HSCs can induce MDSC proliferation in the bone marrow, spleen, and tumor tissue in the HCC mouse model. To further investigate the direct effect of HSCs on MDSCs, BM cells were cocultured with either the HSCs or the H22-activated HSCs for 5 days. The results showed that coculturing with HSCs significantly increased the population of $\mathrm{Gr}^{+} \mathrm{CD}^{+} 1 \mathrm{~b}^{+}$cells from 12.9 to $21.5 \%$, and the percentage of MDSCs was increased to $27.9 \%$ when cocultured with the H22-activated HSCs (Figure 6b).

\section{DISCUSSION}

Our previous studies demonstrated that the activated HSCs could create an immunosuppressive microenvironment in a murine mouse model of subcutaneously injected HCC cells. ${ }^{13}$ However, ectopic tumor cell implantation in the subcutaneous tissues might not mimic the actual microenvironment. 

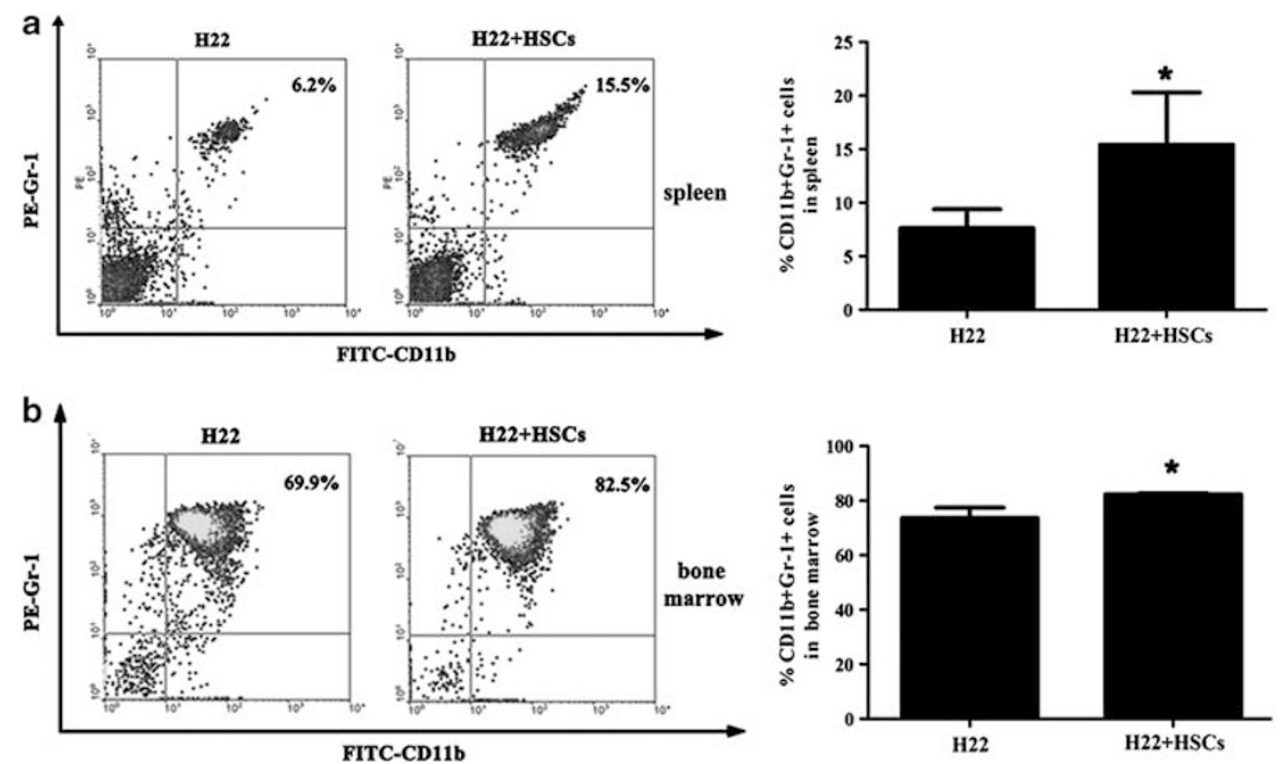

Figure 4 The effect of hepatic stellate cells (HSCs) on the expansion of myeloid-derived suppressor cells. The splenocytes and bone marrow (BM) cells from tumor-bearing mice were isolated for flow cytometric analysis $(n=5)$. The BM cells $(\mathbf{a})$ or splenocytes $(\mathbf{b})$ were double-stained with fluorescently labeled CD11b and Gr-1 mAbs. The data are expressed as the mean \pm s.d. $* P<0.05$.

A growing number of studies from recent years has demonstrated that immune-suppressive cell-mediated immune suppression is one of the most important tumor immune evasion mechanisms and has an important role in the tumor nonresponsiveness. ${ }^{19,20}$ It would be of great interest to investigate the role of HSCs and their interactions with other cells in the tumor environment. An orthotopic implantation HCC mouse model was useful for studying the complex tumor-host interactions and the surrounding microenvironment. So, the cells from the mouse liver cancer cell line, H22, are directly injected into the left lobe of the liver of BALB/c mice to establish the HCC mouse model.

Tregs have a vital role in regulating the immune response and tumor development. The relationship between Tregs and cancer has been extensively investigated, and several independent studies of HCC have demonstrated that Tregs accumulate in the tumors and the peripheral blood of patients and through suppression of anti-tumor immune response promote tumor development. ${ }^{8-10}$ Recent studies demonstrated that Tregs were closely associated with HSCs. Our previous study and those of other researchers have suggested that HSCs induced the expansion of the Treg population $^{14,21,22}$ and LPS could augment this effect. ${ }^{22}$ Ichikawa et al reported that HSCs functioned as regulatory bystanders and promoted Tregs differentiation. ${ }^{23}$ These may represent key players in the mechanism that drives liverinduced tolerance. In this end, we are interested in studying the relationship of HSCs and Tregs in the HCC tumor environment. In this study, we have shown that cotransplantation of HSCs resulted in the increase of $\mathrm{CD} 4{ }^{+}$ Foxp $3^{+}$Tregs in the spleen and tumors. Moreover, cultured activated HSCs induced the expansion of Tregs in a MLR in vitro, which is consistent with our previous reports with HSCs from C57BL6 mice. ${ }^{14}$ In addition, H22-activated HSCs significantly induced the expression Tregs.

MDSCs are another suppressive population of cells and were significantly increased in the peripheral blood and tumors of HCC patients. ${ }^{11}$ As one of the main cell populations that are responsible for regulating the immune responses, MDSCs act to not only suppress the T cell response, but also to induce NK cell anergy ${ }^{24}$ and impair DC function..$^{25}$ In our current studies, there was a significant increase in the

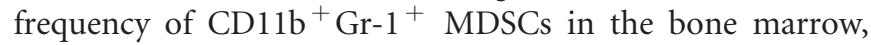
spleen, and tumor of tumor-bearing mice from the HSC co-transplanted group. This demonstrated that the immunomodulatory activity of HSCs in tumor environment was related to the induction of the MDSCs. In addition to the in vivo upregulation of MDSCs by HSCs, the in vitro results also indicated that the HSCs could expand the MDSC population, particularly with the tumor-activated HSCs. We provide a new evidence that HSCs in tumor microenvironment could induce the expansion of the MDSCs population to promote tumor growth. However, in a mouse islet transplant model, co-transplantation of islets with HSCs did not result in more $\mathrm{CD}_{11} \mathrm{~b}^{+} \mathrm{Gr}-1^{+}$cells infiltrating into islet/HSC grafts. ${ }^{18}$ This may be highly relevant for the different mice models.

Previous studies have been shown that the accumulation of MDSCs in tumors was due to tumor-derived factors, which mobilize MDSCs from the bone marrow to the tumor site where they produce multiple MMPs that contribute to tumor invasion. ${ }^{26}$ However, whether activated HSCs in tumor 
a
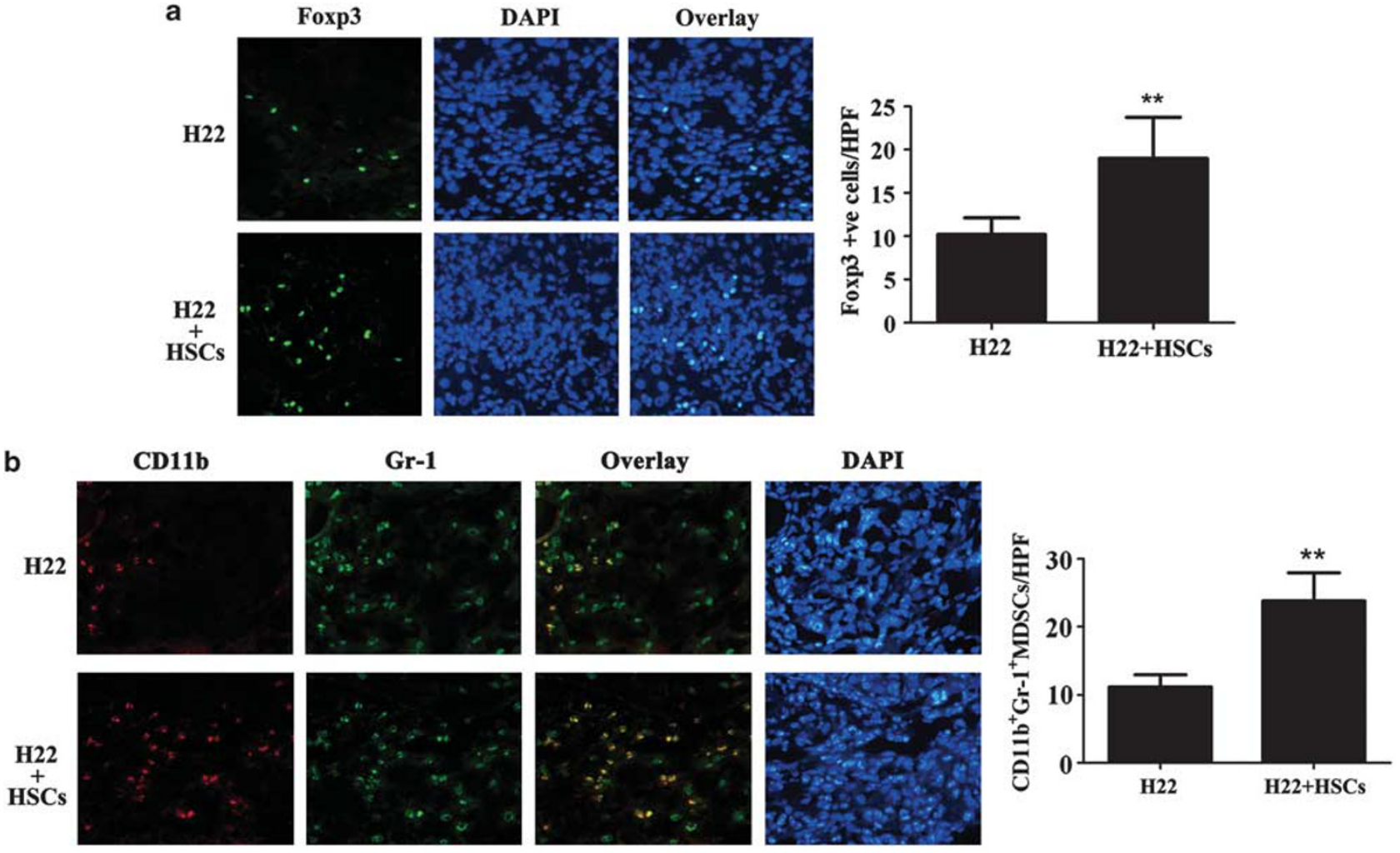

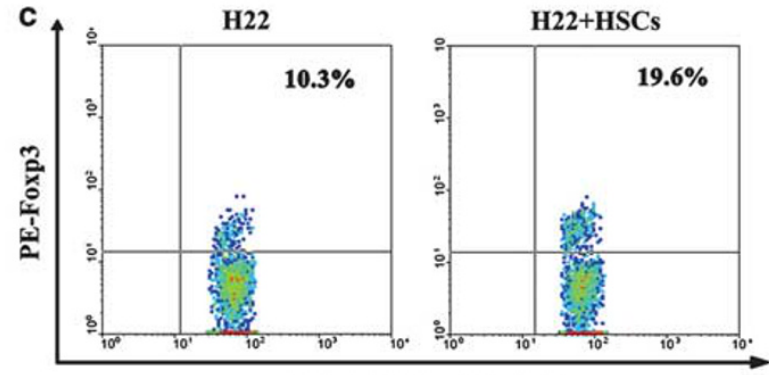

FITC-CD4(CD4 Gated)
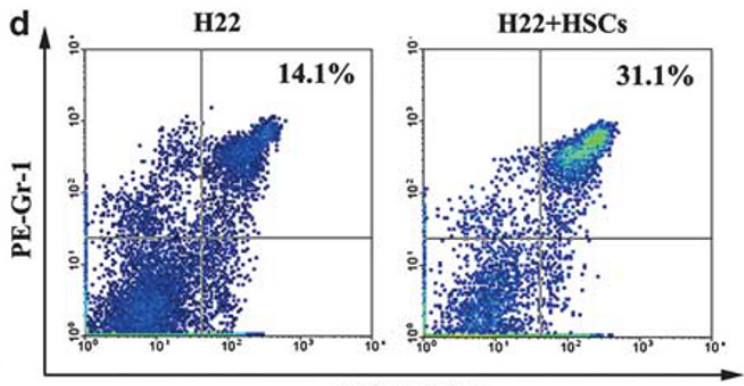

FITC-CD11b

Figure 5 The effect of hepatic stellate cells (HSCs) on the expansion of suppressive cells in the tumor environment. The tumor sample cryosections were analyzed by immunofluorescence staining with Foxp3 (a) and CD11b and Gr-1(green, Gr-1-positive cells; red, CD11b-positive cells) (b). The quantitative data for the immunofluorescence assay are shown as the number of positive or double positive cells per high-power field (HPF). A total of five HPFs were randomly selected for each group. The data are expressed as the mean \pm s.d. (c) The intrahepatic leukocyte (IHL) cells were isolated from the peritumoral tissues and analyzed by flow cytometry. The $\mathrm{CD}^{+}{ }^{+}$populations were gated for analysis. (d) The IHL cells were double-stained with fluorescently labeled CD11b and Gr-1 mAbs and analyzed by flow cytometry. ${ }^{* *} P<0.01$.

microenvironment of HCC could recruit MDSCs require further investigation. MDSCs highly express CCR2, ${ }^{27}$ and CCL2(MCP-1)/CCR2 has a pivotal role in MDSC migration. ${ }^{28}$ Meanwhile, several lines of evidence indicated that HSCs expressed and secreted proteins from the CC chemokine subfamily, including MCP- $1,{ }^{13,29}$ therefore, we hypothesize that HSCs may have a critical role in the recruitment of MDSCs via MCP-1/CCR2 in the HCC tumor environment. A recent study reported that an increase in the frequency of MDSCs was detected in the liver of 4T1 murine breast cancer-bearing mice compared with the control mice, ${ }^{30}$ which further suggested that the existence of activated HSCs in tumor-bearing mice might be the primary mediator in the recruitment and maintenance of MDSCs into the liver.

Our results suggest that a novel role for HSCs in HCC microenvironment and HSCs can prompt HCC progression by enhancement of immunosuppressive cell population. It provides a new mechanistic explanation for the link between HSCs and HCC progression. Targeting HSCs, a new concept in adjuvant immunotherapy, may be introduced to improve the outcome of patient with HCC in near future. 

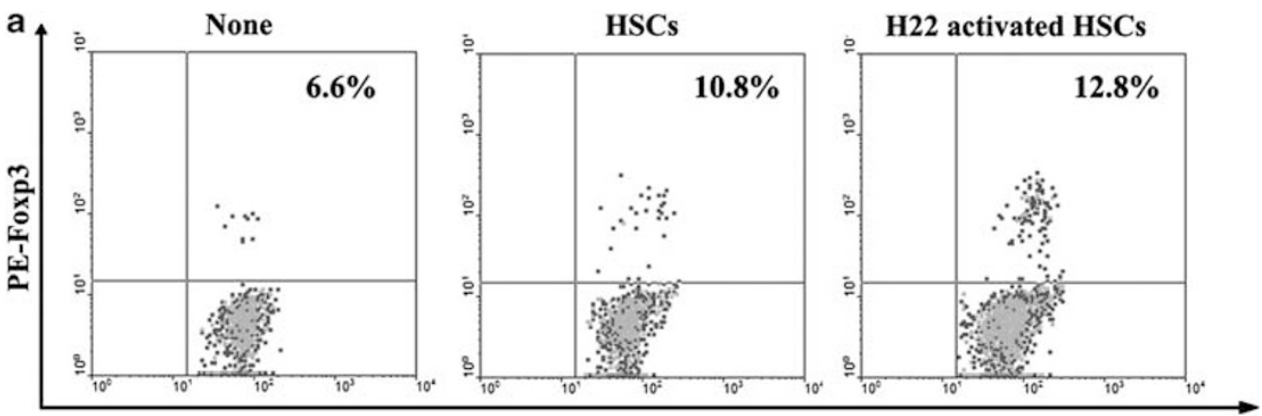

FITC-CD4 (CD4 Gated)
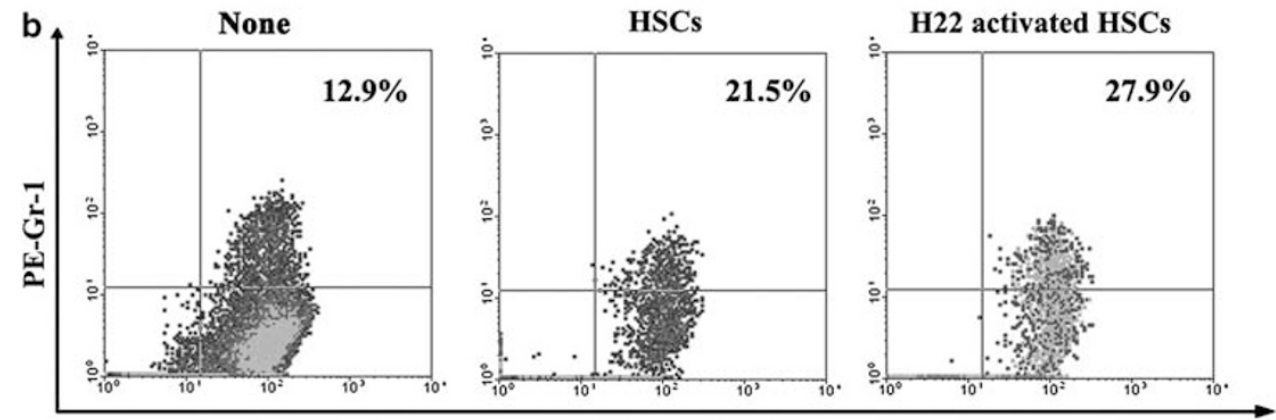

FITC-CD11b

Figure 6 H22-activated hepatic stellate cells (HSCs) induced the expansion of immunosuppressive cells in vitro. (a) The effect of HSCs on regulatory T cells (Tregs) expansion in vitro. Splenic T cells $\left(1 \times 10^{6}\right)$ were cultured with mitomycin-treated dendritic cells (DCs) in the presence of activated HSCs or H22-activated HSCs (T cell/HSC ratio $=40: 1$ ) for 3 days. The cells were double-stained with CD4 and Foxp3 mAb and analyzed by flow cytometry. The number is the percentage of positive cells in the $\mathrm{CD}^{+}$population. The data are representative of three separate experiments. (b) The effect of $\mathrm{HSCs}$ on BM-derived DC propagation in vitro. The BM cells $\left(1 \times 10^{6}\right)$ were cultured with activated $\mathrm{HSCs}$ or $\mathrm{H} 22$-activated $\mathrm{HSCs}(\mathrm{BM}$ cell/HSC ratio $=40: 1)$ for 5 days in the presence of mouse rGM-CSF $(10 \mathrm{ng} / \mathrm{m})$ and IL-4 $(10 \mathrm{ng} / \mathrm{ml})$. The floating cells were double-stained with CD11b and Gr-1 mAbs and analyzed by flow cytometry. The data are representative of three separate experiments.

\section{ACKNOWLEDGMENTS}

We thank Ms Lili Liu and Ms Xiaohong Ma for their help with flow cytometry. This study was supported by grants from the National Key Sci-Tech Special Project of China (No. 2012ZX10002-011-005) and the National Natural Science Foundation of China (No. 81171976 and No. 81201894), and Provincial Natural Science Foundation of Fujian, China (No. 2011D003).

\section{DISCLOSURE/CONFLICT OF INTEREST}

The authors declare no conflict of interest.

1. Budhu A, Forgues $\mathrm{M}, \mathrm{Ye} \mathrm{QH}$, et al. Prediction of venous metastases, recurrence, and prognosis in hepatocellular carcinoma based on a unique immune response signature of the liver microenvironment. Cancer Cell 2006;10:99-111.

2. Chen $\mathrm{CH}$, Kuo $\mathrm{LM}$, Chang $\mathrm{Y}$, et al. In vivo immune modulatory activity of hepatic stellate cells in mice. Hepatology 2006;44:1171-1181.

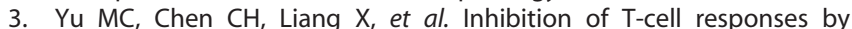
hepatic stellate cells via B7-H1-mediated T-cell apoptosis in mice. Hepatology 2004;40:1312-1321.

4. Hanahan D, Weinberg RA. Hallmarks of cancer: the next generation. Cell 2011;144:646-674.

5. Ha TY. The role of regulatory T cells in cancer. Immune Network 2009; 9:209-235.

6. Filipazzi P, Huber V, Rivoltini L. Phenotype, function and clinical implications of myeloid-derived suppressor cells in cancer patients. Cancer Immunol, Immunother 2012;61:255-263.

7. Facciabene A, Motz GT, Coukos G. T-regulatory cells: key players in tumor immune escape and angiogenesis. Cancer Res 2012;72: 2162-2171.
8. Ormandy LA, Hillemann $\mathrm{T}$, Wedemeyer $\mathrm{H}$, et al. Increased populations of regulatory T cells in peripheral blood of patients with hepatocellular carcinoma. Cancer Res 2005;65:2457-2464.

9. $\mathrm{Fu} \mathrm{J,} \mathrm{Xu} \mathrm{D,} \mathrm{Liu} \mathrm{Z,} \mathrm{et} \mathrm{al.} \mathrm{Increased} \mathrm{regulatory} T$ cells correlate with CD8 T-cell impairment and poor survival in hepatocellular carcinoma patients. Gastroenterology 2007;132:2328-2339.

10. Unitt E, Rushbrook SM, Marshall A, et al. Compromised lymphocytes infiltrate hepatocellular carcinoma: the role of T-regulatory cells. Hepatology 2005;41:722-730.

11. Hoechst B, Ormandy LA, Ballmaier $M$, et al. A new population of myeloid-derived suppressor cells in hepatocellular carcinoma patients induces CD4(+)CD25(+)Foxp3(+) T cells. Gastroenterology 2008; 135:234-243.

12. Ostrand-Rosenberg S, Sinha P. Myeloid-derived suppressor cells: linking inflammation and cancer. J Immunol 2009;182:4499-4506.

13. Zhao W, Zhang L, Yin Z, et al. Activated hepatic stellate cells promote hepatocellular carcinoma development in immunocompetent mice. Int J Cancer 2011;129:2651-2661.

14. Zhao W, Su W, Kuang P, et al. The role of hepatic stellate cells in the regulation of T-cell function and the promotion of hepatocellular carcinoma. Int J Oncol 2012;41:457-464.

15. Semela D, Dufour JF. Angiogenesis and hepatocellular carcinoma. J Hepatol 2004;41:864-880.

16. Banerji S, Ni J, Wang SX, et al. LYVE-1, a new homologue of the CD44 glycoprotein, is a lymph-specific receptor for hyaluronan. J Cell Biol 1999;144:789-801.

17. Solheim JC, Reber AJ, Ashour AE, et al. Spleen but not tumor infiltration by dendritic and $\mathrm{T}$ cells is increased by intravenous adenovirus-Flt3 ligand injection. Cancer Gene Ther 2007;14: 364-371. 
18. Chou HS, Hsieh CC, Yang HR, et al. Hepatic stellate cells regulate immune response by way of induction of myeloid suppressor cells in mice. Hepatology 2011;53:1007-1019.

19. Bianchi G, Borgonovo G, Pistoia V, et al. Immunosuppressive cells and tumor microenvironment: focus on mesenchymal stem cells and myeloid derived suppressor cells. Histol Histopathol 2011;26:941-951.

20. Kim R, Emi M, Tanabe $K$, et al. Tumor-driven evolution of immunosuppressive networks during malignant progression. Cancer Res 2006; 66:5527-5536.

21. Jiang $G$, Yang HR, Wang $L$, et al. Hepatic stellate cells preferentially expand allogeneic CD4 + CD25 + FoxP3 + regulatory T cells in an IL-2-dependent manner. Transplantation 2008;86:1492-1502.

22. Dangi A, Sumpter TL, Kimura S, et al. Selective expansion of allogeneic regulatory $\mathrm{T}$ cells by hepatic stellate cells: role of endotoxin and implications for allograft tolerance. J Immunol 2012;188:3667-3677.

23. Ichikawa S, Mucida D, Tyznik AJ, et al. Hepatic stellate cells function as regulatory bystanders. J Immunol 2011;186:5549-5555.

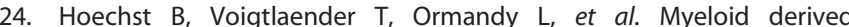
suppressor cells inhibit natural killer cells in patients with hepatocellular carcinoma via the NKp30 receptor. Hepatology 2009;50: 799-807.

25. Hu CE, Gan J, Zhang RD, et al. Up-regulated myeloid-derived suppressor cell contributes to hepatocellular carcinoma development by impairing dendritic cell function. Scand J Gastroenterol 2011:46: 156-164.

26. Ye XZ, Yu SC, Bian XW. Contribution of myeloid-derived suppressor cells to tumor-induced immune suppression, angiogenesis, invasion and metastasis. J Genet Genomics 2010;37:423-430.

27. Gehad $A E$, Lichtman $M K$, Schmults $C D$, et al. Nitric oxide-producing myeloid-derived suppressor cells inhibit vascular e-selectin expression in human squamous cell carcinomas. J Invest Dermatol 2012;132:2642-2651.

28. Huang B, Lei Z, Zhao J, et al. CCL2/CCR2 pathway mediates recruitment of myeloid suppressor cells to cancers. Cancer Lett 2007;252:86-92.

29. Marra F, Romanelli RG, Giannini C, et al. Monocyte chemotactic protein-1 as a chemoattractant for human hepatic stellate cells. Hepatology 1999;29:140-148.

30. Ilkovitch D, Lopez DM. The liver is a site for tumor-induced myeloidderived suppressor cell accumulation and immunosuppression. Cancer Res 2009;69:5514-5521.

(c) (i) (2) (2) This work is licensed under a Creative Commons Attribution-NonCommercial-ShareAlike 3.0

Unported License. To view a copy of this license, visit http:// creativecommons.org/licenses/by-nc-sa/3.0/ 